\title{
Effects of Neonatal Pain, Stress and Their Interrelation on Pain Sensitivity in Later Life in Male Rats
}

\author{
Irina P. Butkevich ${ }^{1,2}$, Viktor A. Mikhailenko ${ }^{1}$, Elena A. Vershinina ${ }^{3}$, and \\ Anna Maria Aloisi ${ }^{4}$ \\ ${ }^{1}$ Department of Ontogenesis of the Nervous System, I.P. Pavlov Institute of Physiology, \\ Russian Academy of Sciences, St. Petersburg 199034, Russia \\ ${ }^{2}$ Department of Normal Physiology, St. Petersburg State Pediatric Medical University, St. \\ Petersburg 194100, Russia \\ ${ }^{3}$ Department of Information Technologies and Mathematical Modeling, I.P. Pavlov Institute of \\ Physiology, Russian Academy of Sciences, St. Petersburg 199034, Russia \\ and \\ ${ }^{4}$ Department of Medicine, Surgery and Neuroscience, University of Siena 53100 Siena, Italy
}

\begin{abstract}
Neonatal pain and stress induce long-term changes in pain sensitivity. Therefore their interrelation is a topical subject of clinical and basic research. The present study investigated the effects of inflammatory peripheral pain and stress of maternal deprivation (MD)-isolation in 1-2- and 7-8day-old Wistar rats (P1,2 and $\mathrm{P} 7,8$ respectively, ages comparable to preterm and full-term human babies) on basal pain and pain sensitivity in conditions of inflammatory pain (formalin test) during adolescence. The neonatal impacts were: pain (formalin-induced, FOR in the paw), stress (a short 60-min MD), or pain+stress combination (FOR+MD), and appropriate controls. We found that stress of short-term maternal deprivation-isolation and inflammatory pain on $P 1,2$ and $P 7,8$ significantly increased the vulnerability of the nociceptive system to inflammatory pain. Maternal deprivationisolation on $\mathrm{P} 1,2$ as compared with a similar impact on $\mathrm{P} 7,8$ had a greater effect on pain sensitivity of the adolescent rats, but the influence of early pain was independent of the injury age. Only adolescent rats with an early combination of pain and maternal deprivation-isolation showed hypoalgesia in the hot plate (HP) test. However licking duration (reflecting pain sensitivity) in these rats did not exceed licking duration in animals exposed only to maternal deprivation-isolation or pain. This study adds new data to the growing body of work demonstrating that early noxious impacts have long-term consequences for the functional activity of the nociceptive system. Our new findings may help to understand the impact of pain and maternal separation in the neonatal intensive care unit.
\end{abstract}

Key Words: adolescence, age of neonatal rats, inflammatory pain, pain sensitivity, stress

\section{Introduction}

Clinical and laboratory findings indicate that repeated neonatal noxious impacts may result in increased sensitivity to future clinical and experimental stimuli $(3,6,13,30,33)$. During the neonatal period, many physiological systems (including the nocicep- tive, endocrine and stress systems) undergo intensive development, with the result that they are especially vulnerable to noxious stimuli $(2,4,18,27,32,33)$. There are different rodent models of long-term effects of neonatal injury on pain processing: repeated needle prick $(3,15)$, neonatal hind paw plantar incision (30), hind paw inflammation with agents such as car-

Corresponding author: Butkevich Irina P., Dr. Sci., Department of Ontogenesis of the Nervous System, I.P. Pavlov Institute of Physiology RAS, Nab. Makarova, 6. 199034, St. Petersburg, Russia. Fax: +7(812)3280501, E-mail: irinabutkevich@yandex.ru Received: November 19, 2015; Revised (Final Version): February 2, 2016; Accepted: February 3, 2016.

(C) 2016 by The Chinese Physiological Society and Airiti Press Inc. ISSN : 0304-4920. http://www.cps.org.tw 
rageenan (CAR), complete Freund's adjuvant (CFA) or formalin $(4,17,20,23,24)$. We will focus on the pattern of change in baseline inflammatory pain thresholds and pain responses to re-inflammation in later life. The results of long-term investigations of the effects of repeated neonatal pain on pain sensitivity have stimulated significant discussion $(6,27,33)$. In most studies, animals with early injuries showed hypoalgesia, a widespread whole body baseline inhibition of sensory and nociceptive thresholds, and hyperalgesia in the presence of ongoing inflammation $(4,23)$. However hypoalgesia was not found in other studies (24). There are reasons to assume that different mechanisms underlie these two opposite displays of early pain $(4,23)$. Hypoalgesia, which presents regardless of the segmental level, is associated with more widespread mechanisms beyond the somatotopically organized nociceptive pathways of the dorsal horn, also involving the brainstem descending pain control system and the hypothalamic-pituitary-adrenal (HPA) system, whereas hyperalgesia involves sensitization of peripheral nociceptors and dorsal horn nociceptive neurons $(23,27)$. The use of various chemical agents to induce early injury in rodents of different neonatal age and the dual (pain/stress) nature of neonatal noxious impacts may influence the long-term alterations in pain sensitivity. Although the sensitive period in regard to such long-term alterations is limited to the first 7-10 days of life in the rat (23), the detection of a more precise age in neonatal rats and the study of the effects of pain and stress interactions in these animals would contribute to a better understanding of this important topic. We used 1-2and 7-8-day-old rats ( $\mathrm{P} 1,2$ and $\mathrm{P} 7,8$ respectively) since the functional activities of the nociceptive system (5, $10)$ and the HPA axis (25) are different in rats of these ages. The rationale of our choice for these two ages is justified by literature data indicating that the level of development of the newborn rat brain is similar to that of a 24-week premature human baby while 7-dayold rat pups have the maturity of a full-term neonate (2). It can be assumed that the impact of early pain and stress of maternal deprivation at these different neonatal ages may influence differently pain sensitivity in the adolescent rats. Adolescence, like the neonatal period, is particularly sensitive to adverse events. The first onset of many stress-related psychopathologies, such as anxiety and depression, usually occurs in adolescence. Therefore, the investigation of stress reactivity characteristics in adolescent individuals with neonatal injuries is particularly important.

The aim of the present study was to investigate the effects of stress and/or inflammation-induced peripheral pain in neonatal male rats of different ages (on P1 and repeatedly on P2 or on P7 and repeatedly on $\mathrm{P} 8$ ) on the basal nociceptive sensitivity and pain sensitivity to inflammatory impact when the same rats are adolescents.

\section{Materials and Methods}

Animals

Wistar rats were obtained from the vivarium of the I.P. Pavlov Institute of Physiology (Koltyshi, Russia). The animals were mated (a male and three females in one cage) and pregnant rats were initially housed four per cage and then individually after the 17 th day of pregnancy. The day of birth was designated as day 0 of life. The animals were maintained under standard conditions, with food and water available ad libitum. On postnatal day 1 the litters were culled to 8 pups per dam with an equal ratio of males and females if possible. Only male rats were used in this study. All experiments were performed in accordance with the European Community Council Directive of November 24, 1986 (86/609/EEC).

\section{Experimental Design}

There were four groups of rats exposed to the impacts on $\mathrm{P} 1$ and repeatedly on $\mathrm{P} 2$ and four groups exposed to the same impacts on $\mathrm{P} 7$ and repeatedly on P8. The impacts were: formalin-induced pain (FOR) and immediate separation from the nest and the dam (maternal deprivation (MD)-isolation) (FOR+MD), (P1,2 n = 11, P7,8 n = 9, from 4 nests); only FOR, after which the pups were immediately returned to the nest and the dam $(\mathrm{P} 1,2 \mathrm{n}=10, \mathrm{P} 7,8$ $\mathrm{n}=10$, from 4 nests); injection of saline (SAL) and immediate maternal deprivation-isolation (SAL+MD), (P1,2 n = 7, P7, $8 \mathrm{n}=9$, from 3 nests); only injection of SAL, after which the pups were immediately returned to the nest and the dam (P1,2 $\mathrm{n}=12, \mathrm{P} 7,8 \mathrm{n}=12$, from 4 nests). In preliminary experiments we found no differences between adolescent rats treated with SAL on $\mathrm{P} 1,2$ or $\mathrm{P} 7,8$ and intact animals (not exposed to any stimuli at an early age) in any of the tested parameters. Therefore the SAL rats were considered the main control group. The formalin concentration $(2.5 \%)$, the volume of formalin or saline $(0.5 \mu 1)$, the site of injection (a single subcutaneous injection into the pad of the left hind paw) and the stress (maternal deprivation, with each pup placed singly in a small cage in a thermostat at $29-33^{\circ} \mathrm{C}$ for $60 \mathrm{~min}$ ) were similar in the $\mathrm{P} 1,2$ and $\mathrm{P} 7,8$ rats. The experimenters were blinded to early life treatment.

At 25 days of age each rat was taken randomly from the nest and subjected to the hot plate test (HP), after which it was labeled using a weak solution of picric acid and returned to the nest to its dam. In $24 \mathrm{~h}$, 
Table 1. Duration of maternal behaviors (pup grooming and nesting) after reunion of pups with the dam

\begin{tabular}{lcrrr}
\hline Groups & \multicolumn{2}{c}{ Pup Grooming (s) (mean \pm SD) } & \multicolumn{2}{c}{ Nesting (s) (mean \pm SD) } \\
\cline { 2 - 3 } & \multicolumn{1}{c}{$\mathrm{P} 1,2$} & $\mathrm{P} 7,8$ & $\mathrm{P} 1,2$ & $\mathrm{P} 7,8$ \\
\hline SAL & $590 \pm 79$ & $630 \pm 77$ & $2576 \pm 98$ & $\mathrm{n}=12$ \\
FOR & $\mathrm{n}=12$ & $559 \pm 78 \pm 97$ & $\mathrm{n}=12$ \\
& $602 \pm 65$ & $\mathrm{n}=10$ & $2564 \pm 96$ & $2584 \pm 101$ \\
SAL+MD & $\mathrm{n}=10$ & $594 \pm 68$ & $\mathrm{n}=10$ & $\mathrm{n}=10$ \\
& $635 \pm 105$ & $\mathrm{n}=9$ & $2399 \pm 119$ & $2563 \pm 102$ \\
FOR+MD & $\mathrm{n}=7$ & $627 \pm 81$ & $\mathrm{n}=7$ & $\mathrm{n}=9$ \\
& $570 \pm 80$ & $\mathrm{n}=9$ & $2490 \pm 107$ & $2378 \pm 116$ \\
& $\mathrm{n}=11$ & $\mathrm{n}=11$ & $\mathrm{n}=9$ \\
\hline
\end{tabular}

Mean time (s) spent by dams grooming pups or nesting in the first hour after reunion with the dam following exposure to formalin (FOR), formalin+maternal deprivation isolation (FOR+MD), saline (SAL) or saline+maternal deprivationisolation (SAL+MD) on P1,2 and P7,8. For the details, see the Results, Maternal behaviors.

pain sensitivity was evaluated in the formalin test in all the rats.

\section{Maternal Behaviors}

Maternal behaviors (pup grooming, self-grooming and nesting time including crouching over pups) were recorded on $\mathrm{P} 1,2$ and $\mathrm{P} 7,8$ for the first $60 \mathrm{~min}$ after reunion in the FOR, FOR $+\mathrm{MD}, \mathrm{SAL}, \mathrm{SAL}+\mathrm{MD}$ groups.

HP Test

Baseline nociceptive sensitivity was assessed using the HP test. The apparatus consists of a $25 \times$ $25 \mathrm{~cm}$ metal $\mathrm{HP}$ surface set at $51^{\circ} \mathrm{C}$, a Plexiglas cage that fits over the HP and a foot-switch timer. Latency to lifting the paw is usually considered an indication of pain threshold. HP latency was averaged from three trials with 10 min intervals between each trial. The testing apparatus was thoroughly cleaned between trials.

\section{Formalin Test}

The injection of dilute formalin into the paw induces the pain response consisting of two phases (8), believed to represent acute and persistent nociception respectively. The pad of the left hind paw was subcutaneously injected with $10.0 \mu \mathrm{l}$ of $2.5 \%$ formalin (7). After injection the rat was placed singly in a chamber $(25 \times 20 \times 10 \mathrm{~cm})$ with transparent glass walls. The licking duration of the injected left hind paw, which reflects pain sensitivity, was recorded during 60 min using a computer program. Each 3-min value of licking duration was averaged and analyzed in the first phase (the first two 3-min periods), the interphase (the third 3-min period) and the second phase (the fourth to twentieth 3-min periods) of the formalin test. The time-course of licking was plotted for each group of rats. Data on licking in FOR, $\mathrm{SAL}+\mathrm{MD}$ and $\mathrm{FOR}+\mathrm{MD}$ rats are presented with respect to SAL unless otherwise indicated.

\section{Statistical Analyses}

The licking data were analyzed using a mixed-model Analysis of variance (ANOVA) with the factors Treatment (SAL, SAL +MD, FOR, FOR+MD), Age of Treatment (P1,2, P7,8) and Time after formalin (within subjects, in the first phase, the first two 3-min periods; the interphase, the third 3-min period; the second phase, the fourth to twentieth 3-min periods). Two-way ANOVA with the factors Treatment (SAL, SAL+MD, FOR, FOR+MD) and Age of Treatment (P1,2, P7,8) was used to analyze the effect of factors on maternal behavior, on HP latency and on the time-course in the formalin test. The least significant difference (LSD) test was used for post-hoc analysis and for testing simple effects when the interactions were significant. The data analysis was carried out with the SPSS Inc. software.

\section{Results}

\section{Maternal Behaviors}

Two-way ANOVA with factors, Treatment (SAL, SAL+MD, FOR, FOR+MD) and Age of Treatment $(\mathrm{P} 1,2, \mathrm{P} 7,8)$ failed to find the effect of the factors on maternal behavior. Maternal grooming of pups was similar in all the groups (SAL, FOR, SAL+MD, FOR+ $\mathrm{MD})$ and there were no significant differences in nesting time among the groups (SAL, FOR, SAL+MD, FOR + MD) regardless of the age of treatment $(\mathrm{P} 1,2$ 

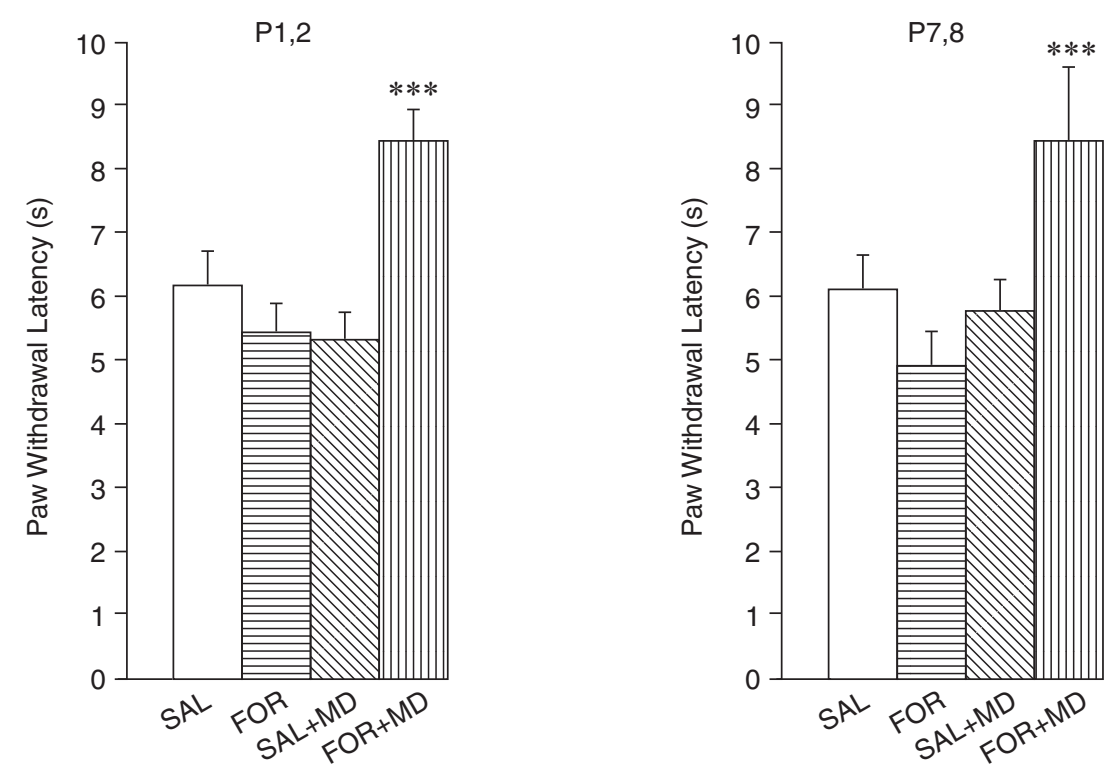

Fig. 1. HP test: latency to thermal paw stimulation (s) in adolescent rats exposed to different experimental conditions on P1,2 or P7,8. For rats, for both ages of treatment, SAL, FOR, SAL+MD vs. FOR+MD, ${ }^{* * *} P<0.001$. Abbreviations: SAL, FOR, injection of saline or formalin respectively on P1,2 or P7,8; SAL $+\mathrm{MD}, \mathrm{FOR}+\mathrm{MD}$, injection of saline and maternal deprivation, injection of formalin and maternal deprivation respectively. Data are mean $\pm \mathrm{SEM}$.

or P7,8) (Table 1).

\section{HP Test}

Two-way ANOVA with the factors Age of Treatment and Treatment revealed a significant effect of Treatment $[\mathrm{F}(3,132)=12.6, * * * P<0.001]$. In rats of both treatment ages, the values were higher in FOR+ MD than in SAL, FOR and SAL+MD $(* * * P<0.001)$ (Fig. 1).

\section{Formalin Test}

Mixed ANOVA was performed with the factors Age of Treatment (P1,2, P7,8), Treatment (SAL, SAL+ $\mathrm{MD}, \mathrm{FOR}, \mathrm{FOR}+\mathrm{MD}$ ) and Time (within subjects). In the first phase there was a significant effect of Age of Treatment $[\mathrm{F}(1,146)=5,2, * P<0.05]$, Treatment $[\mathrm{F}(3,146)=4,3, * * P<0.01]$ and Time $[\mathrm{F}(1,146)=$ $70,1, * * * P<0.001]$, as well as a significant Age of Treatment*Treatment interaction $(P<0.05)$. In the second phase (12-60 min period) there was a significant effect of Treatment $[\mathrm{F}(3,146)=12.97, * * * P<$ $0.001]$ and Time $[\mathrm{F}(16,131)=68.7, * * * P<0.001]$, as well as a significant Time* Treatment interaction $[\mathrm{F}(48,390)=2.4, * * * P<0.001]$.

The post-hoc analysis revealed an increase in licking duration in $\mathrm{SAL}+\mathrm{MD}$ rats exposed on $\mathrm{P} 1,2$ in the first $(* * * P<0.001$ and $* * P<0.01)$ and the second $\left(* P<0.001,{ }^{*} P<0.01, * P<0.05\right)$ phases (Fig. $2 \mathrm{~A}$ ) and in SAL+MD rats exposed on $\mathrm{P} 7,8$ in the sec- ond phase $\left({ }^{++} P<0.01\right)$ (Fig. 2A). Age differences (between P1,2 and P7,8) were found in the first phase $[\mathrm{F}(1,146)=5,2, \wedge P=0.023]$ and at the peak of the second phase $[\mathrm{F}(1,146)=4,3, \wedge P=0.04]$ and $[\mathrm{F}(1,146)$ $=7,8, \wedge P=0.006]$ (Fig. 2A). Licking duration increased in FOR rats exposed on $\mathrm{P} 1,2$, in the first $(* * * P<0.001$ and $* P<0.05)$ and the second $(* P<$ 0.05 and $* * P<0.01$ ) phases (Fig. 2B) and in those exposed on $\mathrm{P} 7,8$, in the first $\left({ }^{++} P<0.01\right)$ and the second $\left({ }^{+} P<0.05,{ }^{++} P<0.01\right.$, and $\left.{ }^{+++} P<0.001\right)$ phases. In FOR + MD animals exposed on $P 1,2$, licking duration increased in the second phase $(* P<0.05)$ and in those exposed on P7,8, in the first phase $\left({ }^{+} P<\right.$ $0.05)$ (Fig. 2C). Licking duration was higher in $\mathrm{SAL}+$ MD than in FOR+MD rats in the first phase $\left({ }^{\#} P<0.05\right)$ and the second phase ${ }^{\# \#} P<0.01$, the fifth, ${ }^{\#} P<0.05$, the sixth and eleventh 3-min periods) in P1,2 animals (Fig. 2, A and C, for comparison). There were no significant changes in the interphase (the third 3-min period) in rats of all groups.

\section{Discussion}

This study is the first to examine the effects of early repeated inflammatory peripheral pain or shortterm MD-isolation or their combination at different neonatal ages of the rat (on P1 and repeatedly P2, or on P7 and repeatedly P8) on pain sensitivity to similar inflammation when the rats are adolescents. The findings indicate that both MD-isolation and pain significantly increased (compared to controls) the 
A

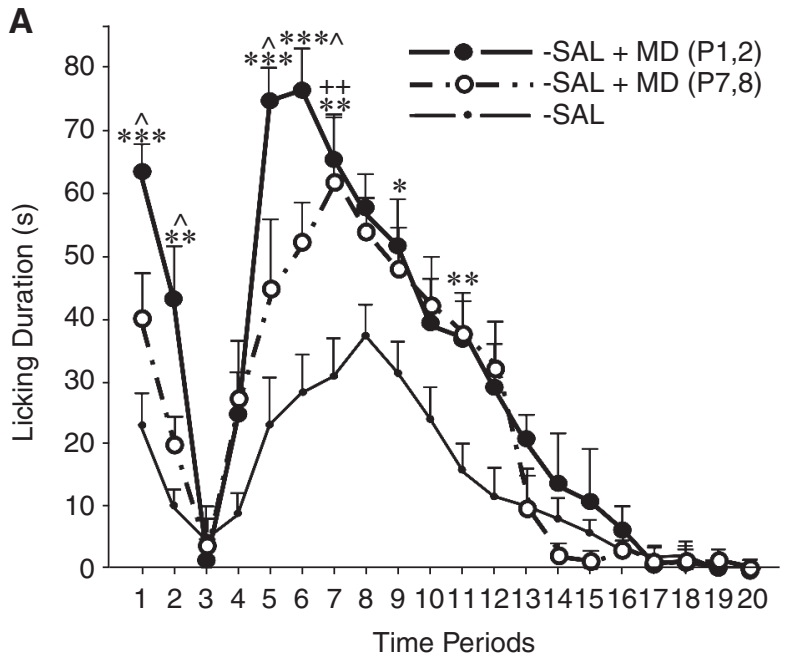

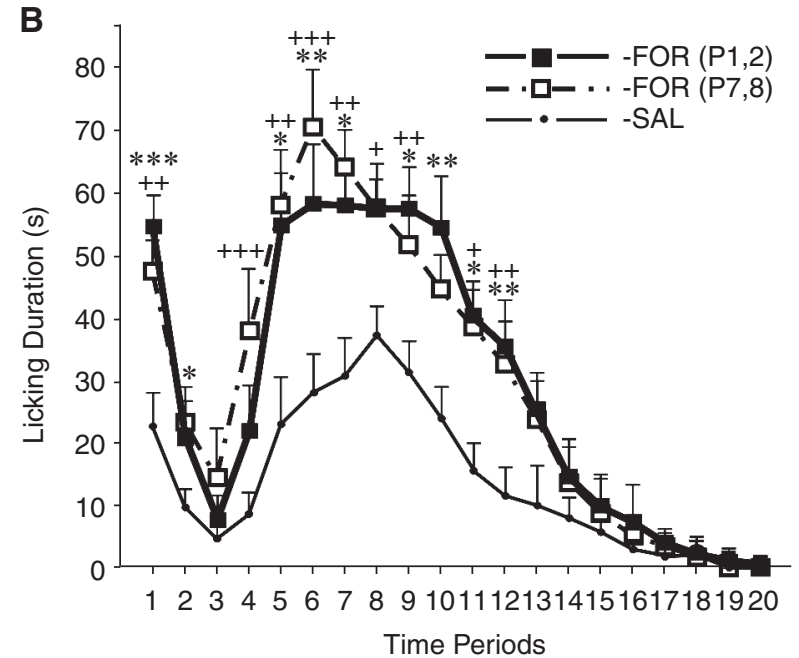

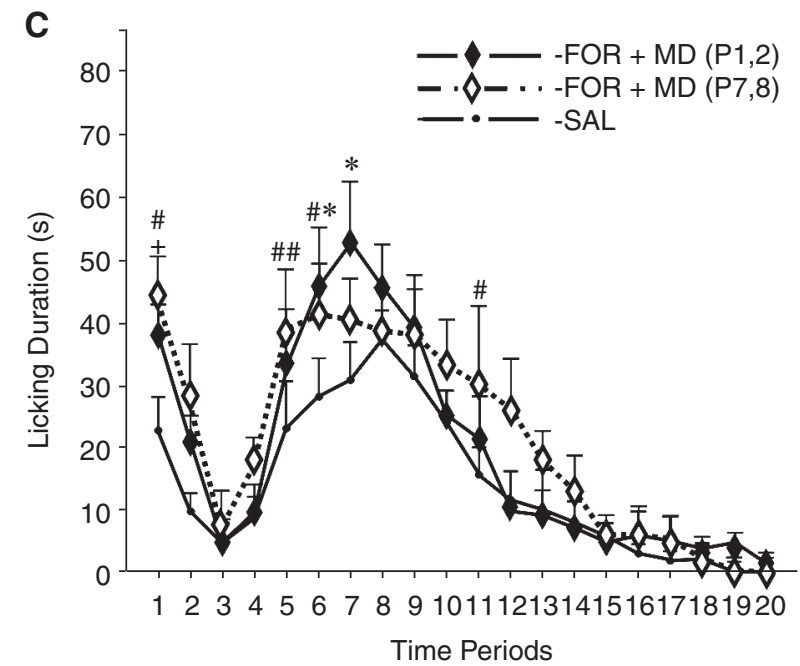

Fig. 2. Time-course of licking behavior in the formalin test in adolescent rats exposed to different experimental conditions on $\mathrm{P} 1,2$ or P7,8 as compared with that in control (SAL) rats. (A) SAL+MD, (B) FOR, (C) FOR+MD. $* P<0.05, * * P<0.01, * * * P<$ $0.001 \mathrm{FOR}, \mathrm{SAL}+\mathrm{MD}, \mathrm{FOR}+\mathrm{MD} v s$. SAL in rats exposed to these experimental conditions on $\mathrm{P} 1,2 ;{ }^{+} P<0.05,{ }^{++} P<0.01$, ${ }^{+++} P<0.001 \mathrm{FOR}, \mathrm{SAL}+\mathrm{MD}, \mathrm{FOR}+\mathrm{MD} v s$. SAL in rats exposed to these experimental conditions on $\mathrm{P} 7,8 ;{ }^{\#} P<0.05,{ }^{\# \#} P<$ $0.01, \mathrm{SAL}+\mathrm{MD} v s$. FOR $+\mathrm{MD}$ (A and $\mathrm{C}$ for comparison) in rats exposed to these conditions on $\mathrm{P} 1,2 ;^{\wedge} P<0.05,{ }^{\wedge} P<0.01$, SAL+MD rats exposed to these conditions on P1,2 vs. on P7,8 (A).

vulnerability of the adolescent nociceptive system in the formalinMD-isolation on P1,2 icreased pain sensitivity in adolescent rats significantly more than a similar impact on $\mathrm{P} 7,8$, whereas early pain increased pain sensitivity regardless of the age at pain injury. Unexpected results were obtained in the adolescent rats exposed to a combination of early pain and MD-isolation regardless of age, their pain response did not exceed that of the rats exposed to early pain or stress of maternal deprivation-isolation (indeed the response of the latter rats was even greater).

We used for the first time short-term MDisolation as a stress impact in contrast to the commonly used long maternal separation $(21,22)$. Our data indicate that neonatal short-term maternal deprivation-isolation increases the vulnerability of the nociceptive system to acute and inflammatory tonic pain when the animals are adolescents to a greater extent in rats exposed to this impact on $\mathrm{P} 1,2$ than on $\mathrm{P} 7,8$. According to the literature, maternal care of pups could reduce noxious impact and result in a beneficial effect on the nociceptive system (17). However we failed to observe any greater care given by the dams to pups of any one of the groups. We assume that most probably the absence of access to the nest, dam and littermates on P1,2 could increase the probability of an HPA stress reaction in the animals, with unfavorable long-term consequences on brain development (14), including areas involved in nociception. In newborn rats the HPA axis can respond to stress by a rise 
in the level of corticosterone, since the stress hyporesponsive period (SHRP) of the HPA only begins on P4 (25). An elevated level of corticosterone in response to stress can influence the development of the stress system and later lead to changes in the response to FOR. The literature data show that early-life stress in rats both decreases the nociceptive threshold and enhances inflammatory mediator-induced hyperalgesia in adults (12). Interestingly, neonatal handling provides resiliency in the stress hormone response to stressors in the adult which can be associated with changes in several neurotransmitter systems within the central nervous system (CNS) (1).

In contrast to the effect of neonatal stress, the consequences of early inflammatory pain did not depend on the age of pain injury, since an increase in licking duration was similar in the adolescent $\mathrm{P} 1,2$ and $\mathrm{P} 7,8$ rats. The increase may be associated with peculiarities of the functioning of the serotoninergic and opiodergic systems and sex hormones at these ages or with the reaction of adolescent rats to pain/stress in the formalin test. It should be noted that clinical studies in humans have demonstrated greater pain sensitivity in preterm neonates than in full-term ones who had required neonatal intensive care $(13,30)$.

Interestingly, hypoalgesia in the HP test was found in rats exposed to FOR+MD on both $\mathrm{P} 1,2$ and $\mathrm{P} 7,8$, but neither early pain nor maternal deprivation alone evoked hypoalgesia in the adolescent rats. Probably different early impacts can differently affect the development of the brainstem descending pain control system. The impact of early injury on the brainstem descending pain control system requires further investigation (6). It is believed that hypoalgesia forms a backdrop to local enhancement of pain sensitivity, and baseline hyposensitivity emerges in 4-5-week-old rats $(17,23)$. In our experimental conditions, 25-day-old FOR+MD rats already revealed hypoalgesia. However our data obtained after the HP test, in the formalin test, showed that the formalininduced pain response in adolescent FOR+MD rats was not higher than that in rats exposed only to early pain or maternal deprivation. This interesting phenomenon, the lesser pain response in adolescent animals with the greater overall neonatal impact, may be associated with the effect of the neonatal and adolescent stress experience interaction ("match-mismatch" hypothesis) (26) and is worthy of attention for the investigation of hypo- and hyperalgesia mechanisms following early impacts.

Neonatal injury is known to increase the strength of descending inhibition from the rostroventromedial medulla (34) and to modulate the endogenous opioidergic tone in the adult CNS (17). Although definite mechanisms of adverse influences in early life on nociceptive pathways are not known, they could be mediated by the HPA axis, which affects the brainstem descending pain control systems and thus influences neurophysiological mechanisms underlying the perception of pain (27). A rodent model of early life pain suggests that inflammatory pain experienced on the day of birth significantly decreases adult somatosensory thresholds and responses to stress- and pain-provoking stimuli and alters regulation of the HPA axis in part through permanent upregulation of central endogenous opioid tone. The nociceptive and HPA systems are in a state of excessive developmental plasticity early in postnatal life and they work in concert to respond to noxious stimuli $(28,29)$.

We cannot compare our results with the available literature data since only the influence of much more prolonged maternal deprivation has been studied on different types of behavior or HPA axis development $(21,22)$. Data on links between early maternal separation and pain sensitivity are very inconsistent. A recent meta-analysis reported that early maternal separation causes a significant overall decrease in sensitivity to nociceptive thermal stimulation involving HP and/or tail-flick tests in rodent offspring (9). The review underscored important sampling and methodological considerations that can effect associations between maternal separation and pain sensitivity.

In our conditions, the rats were subjected not only to short-term maternal deprivation but, for the first time, also to isolation from the littermates and the nest. It is believed that gonadal hormones can, at least partially, determine the effect of glucocorticoids on various systems, since they modulate HPA axis functions throughout ontogeny $(11,19)$.

Moreover, clinical findings demonstrate that a long-term association between early pain-related stress and cortisol are mediated by a genetic variant, suggesting possible involvement of stress/inflammatory mechanisms in HPA programming, at least in boys born very preterm (13). It was underlined in a recent review that an important topic for future investigation is the complex interactions between stress and pain during early life and how these interactions shape nociceptive processing throughout development (33). It is important also to investigate early life noxious impacts on pain sensitivity of females. The present data obtained on male rats is in line with the work performed on rats of different sexes (16). The authors demonstrated that nociceptive influences of repetitive needle pricks during the first postnatal week have a specific effect in inflammatory mechanical hypersensitivity in male rats later in life.

Our study presents new evidence that inflammatory peripheral pain and short maternal deprivationisolation in the neonatal period of the rat (at ages 
comparable to preterm and full-term human babies) increase pain sensitivity to peripheral inflammatory pain during adolescence. Hence, this study suggests that preterm infants may be more vulnerable to maternal isolation than full-term infants. The data on the effects of a combination of two early impacts raise issues concerning the influence of pain and stress interactions in preterm and full-term infants on their pain sensitivity in later life. This study adds new results to the growing body of work demonstrating that early noxious impacts have long-term consequences for the functional activity of the nociceptive system.

\section{Acknowledgments}

This study was supported by the Russian Foundation for Basic Research (grant no. 14-04-00106-a) and a grant from the University of Siena.

\section{References}

1. Alvarez, P., Levine, J.D. and Green, P.G. Neonatal handling (resilience) attenuates water avoidance stress induced enhancement of chronic mechanical hyperalgesia in the rat. Neurosci. Lett. 591: 207-211, 2015.

2. Anand, K.J.S. Physiology of pain in infants and children. Ann. Nestle 57: 7-18, 1999.

3. Anand, K.J.S., Coskun. V., Thrivikraman, K.V., Nemeroff, C.B. and Plotsky, P.M. Long-term behavioral effects of repetitive pain in neonatal rat pups. Physiol. Behav. 66: 627-637, 1999.

4. Anseloni, V.C.Z., He, F., Novikova, S.I., Turnbach Robbins, M., Lidow, I.A., Ennis, M. and Lidow, M.S. Alterations in stressassociated behaviors and neurochemical markers in adult rats after neonatal short-lasting local inflammatory insult. Neuroscience 131: 635-645, 2005.

5. Barr, G.A. Maturation of the biphasic behavioral and heart rate response in the formalin test. Pharmacol. Biochem. Behav. 60: 329-335, 1998

6. Beggs, S. Long-term consequences of neonatal injury. Can. J. Psychiatry 60: 176-180, 2015.

7. Butkevich, I.P., Mikhailenko, V.A., Vershinina, E.A., Otellin, V.A. and Aloisi, A.M. Buspirone before prenatal stress protects against adverse effects of stress on emotional and inflammatory painrelated behaviors in infant rats: age and sex differences. Brain Res. 1419: 76-84, 2011

8. Capone, F. and Aloisi, A.M. Refinement of pain evaluation techniques. The formalin test. Ann. Ist. Super. Sanita 40: 223-229, 2004.

9. Chen, L. and Jackson, T. Early maternal separation and responsiveness to thermal nociception in rodent offspring: a meta-analytic review. Behav. Brain Res. 299: 42-50, 2016.

10. Fitzgerald, M. Developmental biology of inflammatory pain. Brit. J. Anaesth. 75: 177-185, 1995

11. Goel, N., Workman, J.L., Lee, T.T., Innala, L. and Viau, V. Sex differences in the HPA axis. Compr. Physiol. 4: 1121-1155, 2014.

12. Green, P.G., Chen, X., Alvarez, P., Ferrari, L.F. and Levine, J.D. Early-life stress produces muscle hyperalgesia and nociceptor sensitization in the adult rat. Pain 152: 2549-2556, 2011.

13. Grunau, R.E. Neonatal pain in very preterm infants: long-term effects on brain, neurodevelopment and pain reactivity. Rambam Maimonides Med. J. 4: e0025, 2013.

14. Gunnar, M.R., Brodersen, L., Nachmias, M., Buss, K. and Rigatuso,
J. Stress reactivity and attachment security. Dev. Psychobiol. 29: 191-204, 1996.

15. Knaepen, L., Patijn, J., van Kleef, M., Mulder, M., Tibboel, D. and Joosten, E.A.J. Neonatal repetitive needle pricking: plasticity of the spinal nociceptive circuit and extended postoperative pain in later life. Dev. Neurobiol. 73: 85-97, 2012.

16. Knaepen, L., Patijn, J., Tibboel, D. and Joosten, E.A. Sex differences in inflammatory mechanical hypersensitivity in later life of rats exposed to repetitive needle pricking as neonates. Neurosci. Lett. 516: 285-289, 2012.

17. LaPrairie, J.L. and Murphy, A.Z. Neonatal injury alters adult pain sensitivity by increasing opioid tone in the periaqueductal gray. Front. Behav. Neurosci. 3: 31, 2009.

18. Li, J. and Baccei, M.L. Neonatal tissue injury reduces the intrinsic excitability of adult mouse superficial dorsal horn neurons. Neuroscience 256: 392-402, 2014.

19. McCormick, C.M., Furey, B.F., Child, M., Sawyer, M.J. and Donohue, S.M. Neonatal sex hormones have 'organizational' effects on the hypothalamic-pituitary-adrenal axis of male rats. Brain Res. Dev. Brain Res. 105: 295-307, 1998.

20. Negrigo, A., Medeiros, M., Guinsburg, R. and Covolan, L. Longterm gender behavioral vulnerability after nociceptive neonatal formalin stimulation in rats. Neurosci. Lett. 490: 196-199, 2011.

21. Nishi, M., Horii-Hayashi, N. and Sasagawa, T. Effects of early life adverse experiences on the brain: implications from maternal separation models in rodents. Front. Neurosci. 8: 166, 2014.

22. Pierce, A.N., Ryals, J.M. and Wang, R. Vaginal hypersensitivity and hypothalamic-pituitary-adrenal axis dysfunction as a result of neonatal maternal separation in female mice. Neuroscience 263: 216-230, 2014

23. Ren, K., Anseloni, V., Zou, S.P., Wade, E.B., Novikova, S.I., Ennis, M., Traub, R.J., Gold, M.S., Dubner, R. and Lidov, M.S. Characterization of basal and re-inflammation-associated long-term alteration in pain responsivity following short-lasting neonatal local inflammatory insult. Pain 110: 588-596, 2004.

24. Ruda, M.A., Ling, Q.D., Hohmann, A.G., Peng, Y.B. and Tachibana, T. Altered nociceptive neuronal circuits after neonatal peripheral inflammation. Science 289: 628-631, 2000.

25. Sapolsky, R.M. and Meaney, M.J. Maturation of the adrenocortical stress response: neuroendocrine control mechanisms and the stress hyporesponsive period. Brain Res. 396: 64-76, 1986.

26. Schmidt, M.V. Animal models for depression and the mismatch hypothesis of disease. Psychoneuroendocrinology 36: 330-338, 2011

27. Schwaller, F. and Fitzgerald, M. The consequences of pain in early life: injury-induced plasticity in developing pain pathways. Eur. $J$. Neurosci. 39: 344-355, 2014.

28. Victoria, N.C., Karom, M.C., Eichenbaum, H. and Murphy, A.Z. Neonatal injury alters markers of pain and stress in rat pups. Dev. Neurobiol. 74: 42-51, 2014

29. Victoria, N.C. and Murphy, A.Z. The long-term impact of early life pain on adult responses to anxiety and stress: historical perspectives and empirical evidence. Dev. Neurobiol. 37: 1-13, 2016.

30. Vinall, J. and Grunau, R.E. Impact of repeated procedural painrelated stress in infants born very preterm. Pediatr. Res. 75: 584$587,2014$.

31. Walker, S.M. Neonatal pain. Paediatr. Anaesth. 24: 39-48, 2014.

32. Walker, S.M., Beggs, S. and Baccei, M.L. Persistent changes in peripheral and spinal nociceptive processing after early tissue injury. Exp. Neurol. 275: 253-260, 2016.

33. Walker, S.M., Meredith-Middleton, J., Cooke-Yarborough, C. and Fitzgerald, M. Neonatal inflammation and primary afferent terminal plasticity in the rat dorsal horn. Pain 105: 185-195, 2003.

34. Zhang, Y.H., Wang, X.M. and Ennis, M. Effects of neonatal inflammation on descending modulation from the rostroventromedial medulla. Brain Res. Bull. 83: 16-22, 2010. 